\title{
Correction to: Porphyric Neuropathy: Pathophysiology, Diagnosis, and Updated Management
}

\author{
Mohamed Kazamel $^{1}$ (D) $\cdot$ Robert J. Desnick $^{2} \cdot$ John G. Quigley ${ }^{3}$ \\ Published online: 10 December 2020 \\ (C) Springer Science+Business Media, LLC, part of Springer Nature 2020
}

Correction to: Current Neurolology and Neuroscience Reports (2020) 20: 56

https://doi.org/10.1007/s11910-020-01078-8

The original article contained the following two errors:

1- In reference number 95:

95. •• Sardh E, Rejkjaer L, Andersson DE, Harper P. Safety, pharmacokinetics and pharmocodynamics of recombinant human porphobilinogen deaminase in healthy subjects and asymptomatic carriers of the acute intermittent porphyria gene who have increased porphyrin precursor excretion. Clin Pharmacokinet. 2007;46:335-49. https://doi.org/10.2165/ 00003088-200746040-00006This was a phase-1 trial of givosiran in AIP patients. It showed that sustained reductions in ALAS1 mRNA, ALA, and PBG levels occur after monthly subcutaneus injection of the medication and were associated with significant reduction in the annualized attack rate.

Must be corrected to:

The online version of the original article can be found at https://doi.org/ 10.1007/s1 1910-020-01078-8

Mohamed Kazamel

mkazamel@uabmc.edu

1 Department of Neurology, University of Alabama at Birmingham, 1720 2nd Ave. South, SC271, Birmingham, AL 35294-0017, USA

2 Department of Genetics and Genomic Sciences, Icahn School of Medicine at Mount Sinai, New York, NY, USA

3 Department of Medicine, University of Illinois at Chicago, Chicago, IL, USA
95. • Sardh E, Harper P, Balwani M, Stein P, Rees D, Bissell DM, et al. Phase 1 Trial of an RNA Interference Therapy for Acute Intermittent Porphyria. N Engl J Med. 2019;380:549558. doi: https://doi.org/10.1056/NEJMoa1807838. This was a phase-1 trial of givosiran in AIP patients. It showed that sustained reductions in ALAS1 mRNA, ALA, and PBG levels occur after monthly subcutaneous injection of the medication and were associated with a significant reduction in the annualized attack rate.

2- On page 6 and under Supportive and Symptomatic Treatment:

"Prochlorperazine are ondansetron safe options for nausea and vomiting".

Must be corrected to:

"Prochlorperazine and ondansetron are safe options for nausea and vomiting".

Publisher's Note Springer Nature remains neutral with regard to jurisdictional claims in published maps and institutional affiliations. 